\title{
Assessment of Biochemical Profile in Head and Neck Cancer Patients Receiving Concurrent Cisplatin Chemotherapy
}

\author{
Kirthi Koushik and Janhavi Rao*
}

\author{
M.S. Ramaiah Medical College, Bengalooru, NO. 187, A.G.B Ilnd Stage, Mahalaxmipuram, Bengalooru- \\ 560086, India
}

\begin{abstract}
Electrolyte imbalance specifically hypomagnesaemia and hypocalcemia are known events in patients on chemo therapy. We studied thirty- one patients with head and neck cancer who underwent concurrent chemoradiation. Our study has shown that monitoring of calcium and magnesium are equally important and one needs to keep a close watch to avoid morbidity.

Objectives: To study and follow the serum magnesium and calcium levels at onset, at different dosage levels of cisplatin and after completion of cisplatin therapy for head and neck cancer patients receiving concurrent radiation.

Materials and Method: Serum Calcium and Magnesium levels for thirty-one patients receiving cisplatin based chemotherapy and concurrent radiation were studied. The range of radiation dose was from 56-66 GY/week/30 hrs and cisplatin dose was $40 \mathrm{mg} / \mathrm{m} 2 /$ week. The evaluation was carried out for six consecutive cycles.

There was death of one patient during the study.

Results: The results showed a reduction in serum calcium and magnesium levels over the treatment period $(P=0.005)$ with significant error being $<10^{\wedge}-8$.
\end{abstract}

\section{INTRODUCTION}

Head and neck cancers treatment is always multimodal in nature with combination of radiation and chemotherapy as standard of care in majority. Electrolyte imbalance specifically hypomagnesaemia and hypocalcemia are known events in patients on chemotherapy. There is significant number of studies documenting the evidence of hypomagnesaemia and hypocalcemia after chemotherapy but there are very few studies done on the electrolyte imbalance when both chemotherapy and radiotherapy is going on concurrently for head and neck cancer patients. The idea of radiation alone causing electrolyte imbalance is debatable and not addressed in the present study.

A reduced level of magnesium can manifest as symptoms ranging from fatigue, muscle weakness, muscle cramps, to nystagmus hallucinations, depression and convulsions [2].

A reduced level of calcium on the other hand can cause life threatening complications such as laryngospasm, arrhythmias and others such as oral, perioral parasthesias, positive chronotrophic and negative inotrophic effect.

*Address correspondence to this author at the M.S. Ramaiah Medical College, Bengalooru, NO. 187, A.G.B Ilnd Stage, Mahalaxmipuram, Bengalooru560086, India; E-mail: janhaviajit@gmail.com

ISSN: 1927-7210 / E-ISSN: 1927-7229/19
These effects may adversely affect the progress of treatment leading to interruptions and eventual poorer outcomes.

\section{MATERIALS AND METHODS}

\section{Study Sample}

Patients being treated for head and neck carcinoma with concurrent cisplatintherapy at M.S. Ramaiah hospitals.

\section{Study Design \\ Prospective study.}

\section{Sample Size}

30.

\section{Study Period}

6 months.

\section{Inclusion Criteria}

Head and neck cancer patients receiving radical chemoradiation therapy.

\section{Exclusion Criteria}

Post-operative cases and palliative cases as in palliative cases only one modality is used and dose of radiation is different. 
Patients with hypo magnesia and hypocalcemia at the start of the study

\section{Study Methods}

Patients were informed about the research and their consent taken. Serum calcium and serum magnesium levels were measured prior to the commencement of the treatment by collecting sample through venipuncture. The test was repeated weekly during treatment. Along with that data on patient demographics, diagnosis with stage and weekly dose of cisplatin and radiation was collected.

\section{Statistical Analysis}

All the quantitative variables such as magnesium levels, body weight, hemoglobin levels etc. will be summarized using descriptive statistics such as mean and standard deviation. All the quantitative parameters will be summarized in terms of proportion. The change in mean serum magnesium level before, during and after chemoradiation will be compared for statistical significance using repeated measure of Anova/Friedman's test.

\section{RESULTS}

Calcium

\begin{tabular}{|c|c|c|}
\hline Week & Mean & Standard Deviation \\
\hline \hline 1 & 9.24 & 0.52 \\
\hline 2 & 8.99 & 0.45 \\
\hline 3 & 8.81 & 0.48 \\
\hline 4 & 8.66 & 0.39 \\
\hline 5 & 8.50 & 0.35 \\
\hline 6 & 8.16 & 0.53 \\
\hline
\end{tabular}

Magnesium

\begin{tabular}{|c|c|c|}
\hline Week & Mean & Standard Deviation \\
\hline \hline 1 & 2.06 & 0.26 \\
\hline 2 & 1.93 & 0.21 \\
\hline 3 & 1.88 & 0.22 \\
\hline 4 & 1.81 & 0.21 \\
\hline 5 & 1.72 & 0.20 \\
\hline 6 & 1.61 & 0.21 \\
\hline
\end{tabular}

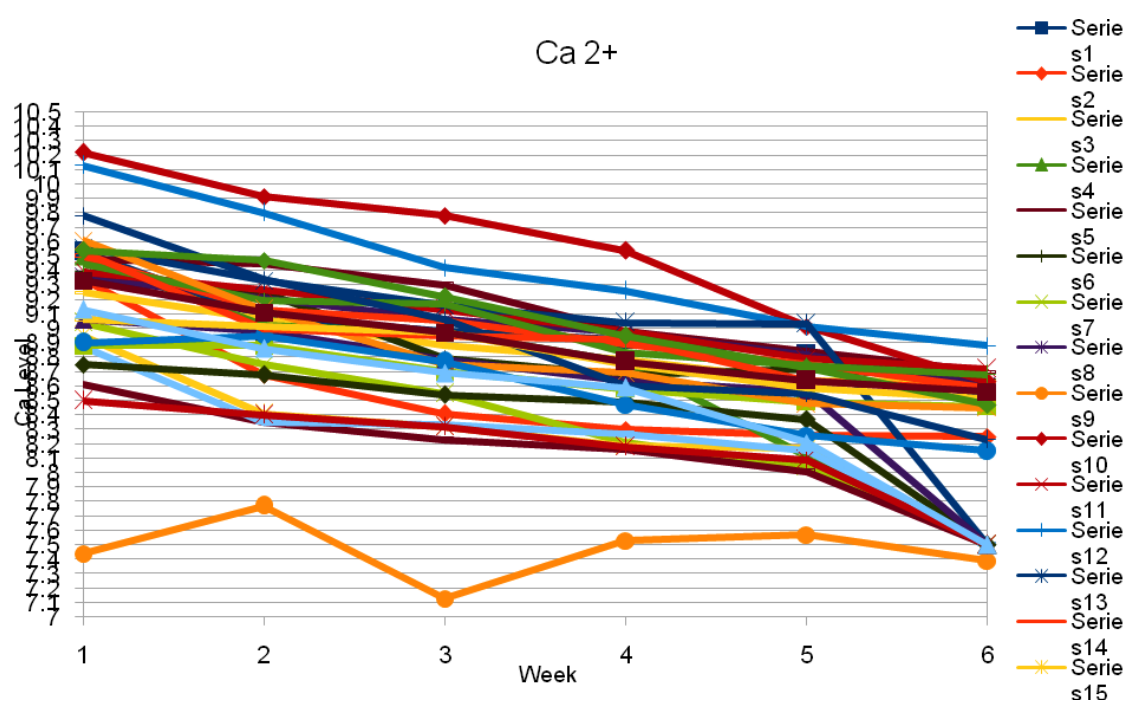




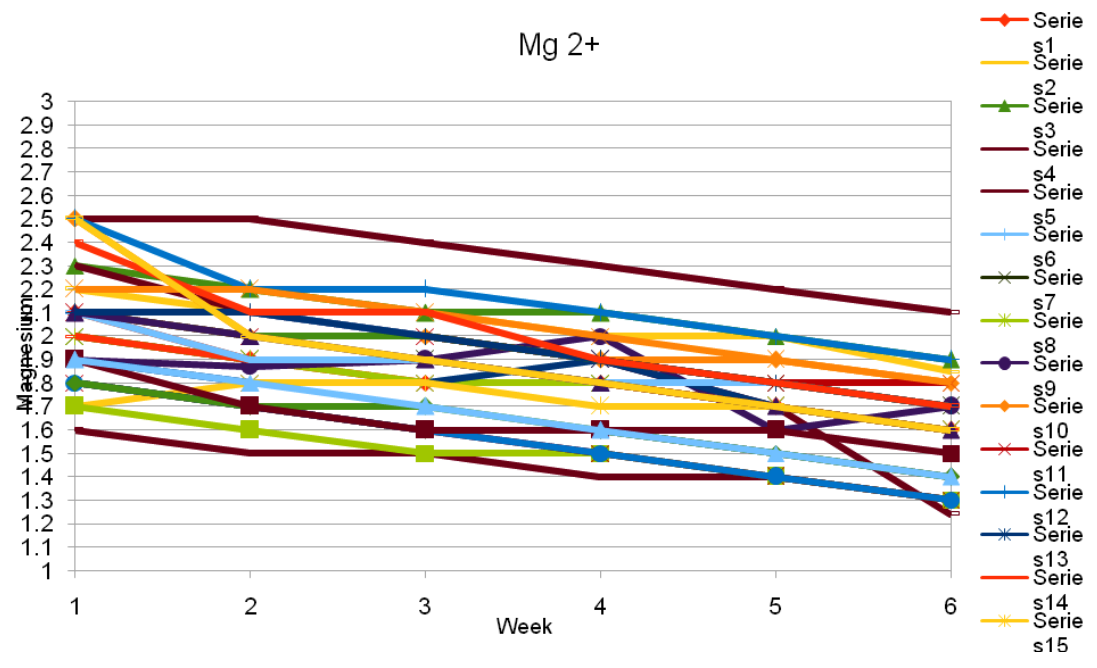

\section{DISCUSSION}

\section{Calcium}

Serum calcium is maintained within a narrow range of $8.5 \mathrm{mg} / \mathrm{dl}$ to $10.5 \mathrm{mg} / \mathrm{dl}$ in the body. The value is influenced by level of Vitamin D3, parathyroid and calcitonin hormone in the body. Vitamin D3 is essential for the intestinal absorption of Calcium. Parathyroid senses the level of calcium and releases more parathyroid which induces increased formation of calcitriol, bone resorption and subsequent release of calcium from the bones. Calcitonin on the other hand is released when there is excess calcium in the serum and it causes increased deposition of calcium in the bone.

Functions of calcium include nerve conduction, neuromuscular and synaptic transmission, muscle contraction, blood coagulation, bone and teeth formation, activation of enzymes, membrane excitability and action as second messenger. Hypocalcemia can manifest in myriad ways such as muscle spasms, carpopedal spasm, facial grimacing, laryngospasm, respiratory arrest, increased intra cranial pressure. There could be mental changes such as irritability, depression and psychosis. Cardiovascular changes can be increased QT interval on ECG, arrhythmias and reduced efficacy of digitalis. Some of the problems are life threatening and can prove fatal.

\section{Magnesium}

Serum Magnesium is regulated within a range of 1.7 $\mathrm{mg} / \mathrm{dl}$ to $2.4 \mathrm{mg} / \mathrm{dl}$ in the body. The value is influenced by renal magnesium reabsorption. Vitamin D3 (Calcitriol) stimulates the intestinal absorption of magnesium. Parathyroid influences the magnesium absorption in the thick ascending limb.

Magnesium participates in many biochemical reactions in the body. It helps maintain normal nerve and muscle function, helps bones remain strong, keeps heart beat steady, regulates blood glucose levels and aid in the production of energy and protein. Hypomagnesemia can cause changes in the neuromuscular junction leading to tetany, tremor, seizures, muscle weakness, ataxia, nystagmus, vertigo, depression, apathy, delirium, psychosis. Cardiac arrhythmias, ECG alterations such as QT or $\mathrm{PR}$ interval prolongation, $\mathrm{T}$ wave flattening or inversion can be seen. Some of the problems are life threatening and can prove fatal.

\section{CONCLUSION}

Our study has shown that calcium and magnesium are equally important in Cisplatin chemotherapy with concurrent radiation and one needs to keep a close watch to avoid morbidity.

\section{RECOMMENDATION}

Serum Magnesium and Calcium levels should be measured routinely in patients receiving concurrent chemoradiation (Cisplatin and Radiation). We recommend that a further study to evaluate the dosage of calcium and magnesium supplementation be undertaken.

\section{REFERENCES}

[1] Stintzing, Sebastian, et al. Clinical relevance and utility of cetuximab-related changes in magnesium and calcium serum levels. Anti-Cancer Drugs 2013; 24(9): 969-974. https://doi.org/10.1097/CAD.0b013e32836411bc 
[2] Katherine M. Hypomagnesemia in the patient undergoing chemotherapy. The One 2013; 8(5): 24-26.

[3] Hodgkinson E, Neville-Webbe HL, Coleman RE. Magnesium depletion in patients receiving cisplatin-based chemotherapy. Clinical Oncology 2006; 18(9): 710-718. https://doi.org/10.1016/j.clon.2006.06.011

[4] Lin, Yu-Jung, et al. Expression of magnesium transporter genes in head and neck cancer patients underwent neoadjuvant cisplatin-based chemotherapy. European Archives of Oto-Rhino-Laryngology 2015; 1-7.

[5] Oka T, Kimura T, et al Magnesium supplementation and high volume hydration reduce the renal toxicity caused by cisplatin-based chemotherapy in patients with lung cancer: a toxicity study. BMC Pharmacol Toxicol 2014; 15: 70. https://doi.org/10.1186/2050-6511-15-70
[6] Hunter, Rodney J, et al. Evaluation of intervention to prevent hypomagnesaemia in cervical cancer patients receiving combination cisplatin and radiation treatment. Supportive Care in Cancer 2009; 17(9): 1195-1201. https://doi.org/10.1007/s00520-008-0574-2

[7] Grădinaru, Irina, et al. Blood plasma and saliva levels of magnesium and other bivalent cations in patients with parotid gland tumors. Magnesium Research 2007; 20(4): 254-258.

[8] Quamme GA, de Rouffignac C. Front Biosci 2000; 5: D694D711. Published online 2000 Aug 1. https://doi.org/10.2741/Quamme

[9] Olhaberry JV, Reyes AJ, Leary WP. S Afr Med J 1983; 63(10): 353-355. https://doi.org/10.1017/S0003581500066579

https://doi.org/10.30683/1927-7229.2019.08.01

(c) 2019 Koushik and Rao; Licensee Neoplasia Research.

This is an open access article licensed under the terms of the Creative Commons Attribution Non-Commercial License (http://creativecommons.org/licenses/by-nc/3.0/) which permits unrestricted, non-commercial use, distribution and reproduction in any medium, provided the work is properly cited. 